\title{
PERCEPÇÃO DAS MULHERES EM SITUAÇÃO DE ABORTAMENTO FRENTE AO CUIDADO DE ENFERMAGEM
}

\author{
Perception of women in abortion situation front of nursing care \\ Percepción de mujeres en abortación con cuidado de enfermeira
}

Lorenna Silva • Enfermeira • Especialista em Saúde da Mulher pela Faculdade Redentor-IDE • Docente na Faculdade de Enfermagem São Vicente de PaulaFESVIP-João Pessoa-PB • E-mail: lorenna.enferm@gmail.com

Nayara Sales • Centro universitário Tabosa de Almeida Aces-Unita-Caruaru-PE Brasil • Enfermeira • E-mail: nayaraalmeidaenf@gmail.com

Renata Santos • Enfermeira Assistencial do Bloco Cirúrgico do Hospital Miguel Arraes/IMIP • Especialista em Clínica Cirúrgica pelo Programa de Residência da Secretaria de Saúde do Estado de Pernambuco/ IMIP E-mail: renata_rivica@outlook.com

Nayale Albuquerque • Doutoranda em Saúde Integral pelo Instituto de Medicina Integral Professor Fernando Figueira • Mestre em Ciências da Saúde/UFPE • Docente do Centro Universitário Tabosa de Almeida Asces-Unita • E-mail: nayalealbuquerque@asces.edu.br

Autora responsável pela correspondência:

Lorenna Silva • E-mail: lorenna.enferm@gmail.com 


\section{RESUMO}

Introdução: $O$ aborto na vida da mulher pode modificar alguns funcionamentos fisiológico e psicológico do seu corpo e desenvolver medo, angústia e solidão. Daí ser fundamental encontrar uma equipe de enfermagem qualificada para recebê-la, de maneira coerente e ética. Objetivo: Identificar o cuidado da enfermagem a partir dos relatos das mulheres em situação de abortamento. Metodologia: Estudo de campo, exploratório, de abordagem qualitativa, realizado no Hospital Jesus Nazareno em Caruaru, estado de Pernambuco, em agosto de 2016. Participaram oito mulheres que receberam a assistência de enfermagem durante o processo abortivo. Considerandose as observâncias éticas da Resolução 466/12 do Ministério da Saúde no Brasil, aplicou-se uma entrevista com as questões norteadoras: "Como você foi recebida no hospital?", "De que forma recebeu a notícia sobre o aborto?", "Como você relata os cuidados da equipe de enfermagem?". Os dados foram transcritos das gravações e interpretados pela análise de conteúdo temática de Bardin. Resultados: Após análise dos dados, emergiram as seguintes categorias: "Acolhimento versus humanização" e "Medicalização na Assistência", revelando a partir das falas das entrevistadas o acolhimento satisfatório desde a chegada até o atendimento final, com resolutividade, correlacionado com os sentimentos vivenciados expressos nos relatos. Conclusões: Diante do exposto e corroborando com o objetivo do trabalho percebeu-se a importância do acolhimento adequado às mulheres na unidade, que ainda pode e deve ser melhorado, a ausência do profissional enfermeiro em todas as etapas deste cuidado, além da medicalização muito presente no modelo do setor da saúde.

Palavras-Chave: Pesquisa qualitativa; Aborto; Enfermagem.

\section{ABSTRACT}

Introduction: Abortion in the life of a woman who is anxiously awaiting the arrival of her child can modify some normal physiological and psychological functioning of her body, especially when she is not expecting this to happen. The predominant feelings of aborted women, mostly spontaneous abortions, are fear, anguish and loneliness. The commitment to welcoming as a form of care is extremely relevant in the nursing team. Objective: To identify nursing care from the discourses of aborted women. Methodology: This is a descriptive exploratory field study with a qualitative approach, carried out at the Jesus Nazareno Hospital, located in the city of Caruaru state of Pernambuco, in August 2016. Eight women participated in the study. facing an abortion process, based on data saturation criteria, and an interview was conducted with the following guiding questions: "How were you received at the hospital?", How did you receive the news about abortion?" and "How did you report the care the nursing staff?". The data obtained from the interviews were organized through transcription of the recordings and analyzed through the application of Bardin's matic content analysis technique. All ethical observances contemplated in solution No. 466/12 / MS. Results: After applying the analysis, the following egories emerged: "Welcome versus humanization" and "Medicalization in sistance". Presenting from the speeches of the interviewees the reception in the 
service correlating with the feelings experienced. Conclusions: Given the above and corroborating with the objective of the study, it was realized the importance of adequate reception to women in the unit, the absence of the professional nurse before this care, in addition to the medicalization present in the health sector model.

Keywords: Qualitative research; Abortion; Nursing.

\section{RESUMEN}

Introducción: el aborto en la vida de una mujer que espera ansiosamente la llegada de su hijo puede modificar el funcionamiento fisiológico y psicológico normal de su cuerpo, especialmente cuando no espera que esto suceda. Los sentimientos predominantes de las mujeres abortadas, en su mayoría abortos espontáneos, son miedo, angustia y soledad. El compromiso con la atención como forma de atención es extremadamente relevante en el equipo de enfermería. Objetivo: identificar los cuidados de enfermería a partir de los discursos de las mujeres abortadas. Metodogía: Estudio exploratorio de campo descriptivo con enfoque cualitativo, realizado en el Hospital Jesús Nazareno, ubicado en la ciudad de Caruaru, estado de Pernambuco, en agosto de 2016. Ocho mujeres participaron en el estudio. frente a un proceso de aborto, basado en criterios de saturación de datos, y se realizó una entrevista con las siguientes preguntas orientadoras: “¿Cómo fue recibido en el hospital?", “¿Cómo recibió las noticias sobre el aborto?" y “¿Cómo lo recibió? ¿Informar el cuidado del personal de enfermería?". Los datos obtenidos de las entrevistas se organizaron mediante la transcripción de las grabaciones y se analizaron mediante la aplicación de la técnica de análisis de contenido temático de Bardin. Se consideraron todas las observancias éticas contempladas en la Resolución N ${ }^{\circ} 66 / 12$ / MS. Resultados: Después de aplicar el análisis, surgieron las siguientes categorías: "Bienvenida versus humanización" y "Medicalización en asistencia". Presentando a partir de los discursos de los entrevistados, la recepción en el servicio se correlaciona con los sentimientos experimentados. Conclusiones: dado lo anterior y corroborando con el objetivo del estudio, se dio cuenta de la importancia de una recepción adecuada para las mujeres en la unidad, la ausencia de la enfermera profesional antes de esta atención, además de la medicalización presente en el modelo del sector de la salud.

Palabras clave: investigación cualitativa; Aborto Enfermería 


\section{Introdução}

O acontecimento de um aborto na vida da mulher que espera ansiosamente pela chegada do seu filho pode modificar alguns funcionamentos normais do seu corpo, tanto fisiológico quanto psicológico, principalmente quando ela não está esperando que esse fato aconteça. O aborto, caracterizado pela Organização Mundial de Saúde (OMS), "é a interrupção da gestação após a fecundação do ovócito entre a $20^{\mathrm{a}}$ e $22^{\mathrm{a}}$ semana completa e peso até 500 gramas"1.

Segundo Aquino², os sentimentos predominantes das mulheres em situação de abortamento, em sua grande maioria abortos espontâneos, são o medo, angústia e solidão. Muitas mulheres estão em processo de abortamento e não reconhecem os sinais e sintomas, gerando medo e angústia diante da possibilidade de perda do bebê, e a maioria se encontra sozinha no momento de início dos sinais e sintomas, chegando a unidade de atendimento esperando um prognóstico que, diante do resultado, gera sentimento de culpa, depressão e revolta.

O compromisso com o acolhimento como forma de cuidado é de extrema relevância na equipe de enfermagem. Pode-se dizer que na hora que aquela mulher chega à unidade hospitalar com seus sentimentos totalmente abalados, é importante encontrar uma equipe de enfermagem bem preparada para recebê-la, de maneira coerente e com ética. O simples gesto de explicar os procedimentos que serão feitos com atenção especial para ela e a explicação de que não haverá nenhuma violação dos direitos humanos, já se torna um acolhimento de qualidade. A postura no qual esse profissional se apresenta, os gestos, as expressões, o tom de voz, tudo isso é percebido pela paciente e são mecanismos de apoio que contribuem na forma terapêutica ajudando-as a superar as angústias, os medos de julgamento, discriminação e sofrimento ${ }^{3}$.

Diante da real necessidade de uma assistência humanizada cabe aos profissionais saúde, especialmente os profissionais de enfermagem, visto que são eles que ntêm um contato mais próximo e contínuo durante a assistência, acolher sem lquer discriminação ou julgamento, executar técnicas e procedimentos e promover s de continuidade da assistência ${ }^{4,5}$. 
Essa pesquisa se mostra relevante considerando-se que a assistência à saúde deva ser disponibilizada igualitariamente para todas as mulheres no ambiente hospitalar (nesse caso da maternidade), independente da sua condição clínica e que as pacientes consigam se sentir acolhidas diante da situação que enfrentam.

Com esta pesquisa pretende-se visualizar de que forma ocorre o cuidado prestado pela equipe de enfermagem, a fim de proporcionar através da literatura formas mais sólida, a possibilidade $\mathrm{da}(\mathrm{o})$ enfermeira(o) planejar ações de melhoramento relacionados com a assistência à saúde da mulher a partir de informações extraídas da ótica e palavras das próprias pacientes quanto a assistência lhes fornecida. Diante deste contexto, o objetivo da pesquisa é identificar o cuidado da enfermagem a partir dos discursos das mulheres em situação de abortamento.

\section{Metodologia}

Trata-se de um estudo de campo, descritivo, exploratório de abordagem qualitativa, realizado no Hospital Jesus Nazareno, maternidade que atende gestação de alto risco, localizado no município de Caruaru-PE. A pesquisa foi realizada no período de agosto a setembro de 2016 após a aprovação de número: 1.590.230 do projeto pelo Comitê de Ética em Pesquisa.

A população de estudo teve como critério de inclusão as mulheres que deram entrada no referido hospital, acometidas pelo processo abortivo, sem discriminação do tipo de aborto (espontâneo ou provocado). Para determinar a quantidade de mulheres avaliadas foi utilizado critério de saturação de dados, que consiste em uma ferramenta usualmente aplicada em pesquisas qualitativas no campo da saúde. É definido como a suspensão de inclusão de novos participantes quando os dados obtidos passam a apresentar, na avaliação dos pesquisadores, certa repetição, não sendo considerado relevante prosseguir com a coleta de dados ${ }^{6}$.

Assim, a coleta de dados ocorreu com 8 mulheres que se encontravam internadas maternidade e foram entrevistadas abordando as seguintes questões: "Como você recebida no hospital", "De que forma você recebeu a notícia?" e "Como você relata cuidados da equipe de enfermagem?". Para os devidos registros, foi utilizado um yador de áudio com o objetivo de capturar detalhadamente a opinião expressada 
em cada resposta, realizada em um ambiente isolado e livre de ruídos para melhor qualidade na obtenção dos dados e para evitar interrupções durante a entrevista. Os dados obtidos com as entrevistas foram organizados por meio da transcrição das gravações, após audição repetida das falas registradas pelo método de Bardin que consiste em três fases: Pré-análise, exploração do material, tratamento dos resultados, que permitirá ao final da análise um resultado estruturado da pesquisa ${ }^{7}$.

\section{Resultados e Discussão}

Observando-se a caracterização das mulheres do estudo, quanto à faixa etária as mesmas encontravam-se entre 13 a 39 anos, em que a escolaridade predominante foi o ensino médio completo, sendo que 3 destas mulheres ainda estão estudando. Em relação à ocupação, apenas 2 mulheres possuíam trabalho remunerado, as demais eram agricultoras, dona de casa ou estavam concluindo os estudos. Quanto à paridade, 3 das mulheres entrevistadas eram nulíparas, enquanto as demais já possuíam de 2 a 3 filhos.

Após a análise realizada, surgiram as seguintes categorias: "Acolhimento versus humanização" e " Medicalização na Assistência".

Categoria 1- Acolhimento versus humanização

As falas revelam que as mulheres foram bem recebidas pelos profissionais que as atenderam. No primeiro contato foi notado que a enfermagem não esteve presente em todas as falas e que algumas mulheres foram recebidas por outros profissionais da unidade.

\footnotetext{
"...Aqui tudo foi muito bom." (E2)

“...fui transferida para aqui, ai aqui me atenderam bem." (E3)

“... Quando eu cheguei, fui bem recebida, tirei minha ficha aí a mulher disse: minha filha tenha calma." (E4)

"Muito bem... fui recebida pelo rapaz da maca, cheguei na emergência e elas já começaram a me atender." (E5)

"Foi bem, me deram atenção..." (E6)
} 
O acolhimento desenha-se na recepção do usuário nos serviços de saúde, desde a sua chegada até o atendimento com resolutividade e co-responsabilização. Acarreta em prestar um atendimento integral, orientando conforme o caso, o usuário e a família, garantindo a articulação com os outros serviços de saúde para a continuidade da assistência quando necessário. $\mathrm{O}$ acolhimento não envolve apenas chamar a mulher pelo seu nome, vai além disso, é de suma relevância a comunicação verbal: ouvir, dar atenção, compreender e solidarizar-se com a mulher e a comunicação não verbal: observar gestos, tom de voz, postura, olhares, entre outras atitudes. Desta forma o acolhimento é, antes de tudo uma postura a ser exercida por toda a equipe, refletindo na qualidade da assistência, quando exercitado de forma eficaz, facilita o processo de trabalho junto aos serviços, pois a escuta do relato desta mulher, vítima do processo abortivo é de suma importância para que ocorram intervenções nos momentos exatos do cuidado ${ }^{8,9}$.

Convém ressaltar a ausência em alguns momentos do profissional enfermeiro no atendimento, além da falta de humanização por parte desse profissional, como observado nas falas abaixo:

“O médico não dá muita atenção, nem as enfermeiras aqui, eles passam vê se a pessoa está com dor. Teve uma que perguntou: está tudo bem? Respondi, estou com cara de quem está bem? Ai ela ficou pra minha cara e disse isso é normal, quem mandou querer ter filho agora..." (E8)

"Eu cheguei, esperei pro médico me atender, quando o médico me atendeu, me encaminhou lá pra cima...Lá em cima foi tudo bom, agora quando chegou aqui embaixo, até agora não está sendo bom." (E7)

O motivo, da ausência do enfermeiro, este associado muitas vezes a influência do sistema de organização de trabalho, como Taylorismo e Fayolismo, no entanto não ustifica a falta da humanização. A organização do modelo de Taylor e Fayol possui aracterísticas particulares como: divisão de tarefas, otimização do tempo, ocorrendo xcessiva preocupação da equipe de enfermagem com manuais de procedimentos, inas, normas, fragmentação da assistência entre outros, fazendo com que a equipe 
preocupe-se em atingir metas impostas pelo serviço, e que desta forma seus desempenhos são avaliados pelo quantitativo de procedimentos realizados ${ }^{15}$.

Para o atendimento humanizado ocorra, requer do profissional segurança, ofertar palavras de conforto, carinho, atenção, deixar que a mulher se expresse, até mesmo que reclame. O cuidado segundo Leonard Boff é mais que um ato, é uma atitude, sendo assim é necessário que os profissionais prestem assistência de forma holística, sem discriminação em relação ao processo abortivo independente de ser induzido de forma legal ou ilegal, ou até espontâneo. Faz-se necessário a compreensão da importância do atendimento holístico de forma subjetiva, não de forma mecânica seguindo apenas protocolos. $\mathrm{O}$ atendimento holístico visa perceber que existe um ser biopsicossocial e espiritual que possui suas singularidades e cabe aos profissionais respeitar esta mulher, assim sabendo intervir de forma coerente e imparcial nas situações que possam ocorrer durante o cuidado ${ }^{12}$.

\section{Categoria 2- Medicalização na assistência}

A terminologia medicalização indica um processo pelo qual o modo de vida dos seres humanos é apropriado pela medicina e que interfere na construção de conceitos, regras, higiene, normas e costumes de uma população, entre outros. A medicalização é evidente em vários setores da saúde, até mesmo no cuidado, podendo gerar problemas em relação a perda da autonomia pelo paciente e do seu próprio autocuidado ${ }^{13}$.

Foi possível observar uma padronização no cuidado em que ocorria a realização de procedimentos mecanizados juntamente com a fragmentação e hierarquização do trabalho, características essas presentes na teoria da administração científica de Frederick W. Taylor (1856-1915). Essa teoria fundamenta-se na aplicação de métodos da ciência positiva, racional e a fim de alcançar a máxima produtividade ${ }^{14}$.

“...Ai peguei e fiz a ultrassom, ai ela disse o coração da criança parou, tem que tirar a criança, fazer a limpagem..." (E4)

“...cheguei na emergência e elas já começaram a me atender e já me deram uma injeção e me encaminharam para coletagem." (E5) 
“...ai ela fez outro toque, mas o colo do útero ainda estava fechado, ai falaram que tinha que ficar internada, me colocaram em uma maca e me levaram lá pra cima, uma sala do lado de cá, eu perguntei: vou ficar fazendo o que aqui? Ela respondeu: você vai pra sala de coleta. Eu perguntei o que é isso? Ela Respondeu:é pra você tirar seu bebê." (E8)

Mediante este cenário o ato médico se caracteriza como repetidor de conhecimentos habilitados pela ciência, assim, retornando ao universo das séries de produção, que marcam a sociedade industrial-tecnológica. É importante ressaltar a relação médico-paciente, considerada uma técnica ou procedimento na prestação do cuidado, que tem como objetivos a personalização da assistência, humanização do atendimento e o direito à informação ${ }^{16}$.

Apresenta-se também como resultado extraído das falas a demora para o atendimento, o tempo de espera das mulheres.

$$
\begin{aligned}
& \text { "...Bom na chegada teve um imprevisto, tinha muita gente pro médico } \\
& \text { atender, esperamos bastante..." (E1) } \\
& \text { "...Cheguei aqui com dor, não estava aguentando a fila, chorando muito, } \\
& \text { com muita dor, estava nem aguentando mais." (E4) }
\end{aligned}
$$

Esses resultados apontam que, a atitude do profissional enfermeiro em voltar-se para abordagens burocráticas, lembrando apenas de cumprir metas, pode levar a não priorização das necessidades do cliente, gerando desmotivação e descontentamento tanto para o cliente, como para o profissional, desta maneira refletindo de forma direta na melhora do quadro clínico ${ }^{17}$.

Desta forma faz-se necessário que os profissionais busquem o aprimoramento contínuo de sua prática para a prestação do cuidado aos clientes cada vez mais embasadas em princípios científicos que contribuam para um cuidado de forma holística. Cuidado este que deve respeitar as particularidades de cada cliente fazendonecessário que o profissional enfermeiro ouça, dê atenção, compreenda e se lidarizare com a mulher, observando gestos, tom de voz, postura, olhares, entre ras atitudes, que possam influenciar na sistematização do cuidado, e acima de tudo 
prestar assistência equânime, sem discriminação, seja esta de causa social, cultural, racial e financeira ${ }^{12,16}$.

\section{Conclusões}

A partir dos resultados expostos pode-se concluir que existiram diferentes posturas do cuidado de enfermagem, e que apesar desta categoria profissional não estar presente em todas as etapas, as mulheres sentiram-se bem acolhidas, mesmo com a demora no atendimento e forma de execução do trabalho na unidade. Paralelamente algumas não consideraram satisfatório o cuidado recebido.

O acolhimento é um fator de extrema importância proporcionando a vivência do processo abortivo menos traumatizante e mais humano. Cabe também ao enfermeiro e equipe de enfermagem estarem sempre presentes no acolhimento e na humanização, que durante a assistência em alguns relatos foram prestados muitas vezes por diversos profissionais do serviço, como: recepcionista, maqueiro, entre outros.

O cuidado de enfermagem observado pelas falas mostra-se distante do que as políticas preconizam. Outro fator que se questiona é o que as mulheres esperavam ou compreendiam de como deveriam ser os cuidados? Será que houve preparo ou instrução para esse tipo de situação? Essas questões não foram completamente respondidas, o que mostra a necessidade do desenvolvimento de mais estudos acerca do tema, com aprofundamentos que possam contribuir para uma futura mudança na atenção às mulheres em situação de abortamento nas unidades do Brasil.

\section{Referências}

1. Domingos S, Ribeiro F, Merigui MAB. O aborto como causa de mortalidade materna: um pensar para o cuidado de enfermagem. Esc. Anna Nery, 2010; 14(1):177-181.

Aquino EL. Atenção à saúde da mulher em situação de abortamento: experiências mulheres hospitalizadas e práticas de profissionais de saúde [tese]. São Paulo: aculdade de Saúde Pública: São Paulo; 2012.

Soares MCS, Freitas VEO, Cunha ARR, Almeida JLS, Souto CMRM, Dantas RA. áticas de enfermagem na atenção às mulheres em situação de abortamento. Revista Rede de Enfermagem do Nordeste-Rev Rene [periódico na Internet]. 2012;13(1):140- 
http://www.revistarene.ufc.br/revista/index.php/revista/article/view/25.

4. Ministério da Saúde (BR). Atenção Humanizada ao Abortamento. Brasília: Ministério da Saúde. 2011.

5. Cunha ATR; Vilar RLA; Melo RHV; Silva AB; Rodrigues MP. Percepções de usuários sobre humanização na estratégia saúde da família: um estudo ancorado na teoria da dádiva. Revista Ciência Plural, 2018. 4(3):16-31.

6. Fontanella BJB, Ricas J, Turato ER. Amostragem por saturação em pesquisas qualitativas em saúde: contribuições teóricas. Cad. Saúde Pública. 2008;24(Supl 1);1727.

7. Bardin L. Análise de conteúdo. Lisboa: Ed Edições de 70; 1977. p. 31.

8. Brasil. Ministério da Saúde. Secretaria de Atenção à Saúde, Núcleo da Política Nacional de Humanização. Acolhimento nas práticas de produção de saúde. Brasília: Ministério da Saúde, 2006.

9. Brasil. Ministério da Saúde. Secretaria de Atenção à Saúde, Departamento de Ações Programáticas Estratégicas, Área Técnica de Saúde da Mulher. Atenção Humanizada ao Abortamento: norma técnica. Brasília: Ministério da Saúde, 2011.

10. Conselho Federal de Enfermagem. Resolução COFEN-240/2000, de 30 de agosto de 2000. Aprova o Código de Ética dos Profissionais de Enfermagem e dá outras providências [internet]. [acesso em 10 set 2016]. Disponível em: < http://www.cofen.gov.br/resoluo-cofen-2402000-revogada-pelaresoluo- cofen3112007_4280.html>.

11. Mariutti MG, Almeida AM, Panobianco MS. O cuidado de enfermagem na visão de mulheres em situação de abortamento. Rev. Esc. Enfermagem [periódico na internet]. 2014;2(1):50-59. Disponível em:<http:// www.scielo.br/pdf/reeusp/v44n3/08.pdf $>$.

12. Bazotti KDV, Stumm EMF, Kirchner RM. Ser cuidada por profissionais da saúde: percepções e sentimentos de mulheres que sofreram abortamento. Texto \& contexto enferm [periódico na internet]. 2009;18(1):147-54. Disponível em: khttp://www.scielo.br/pdf/tce/v18n1/v18n1a18.pdf>.

13. Foucault M. Microfísica do poder. São Paulo: Edições Graal, 2008.

Matos E, Pires D. Teorias administrativas e organização do trabalho: de Taylor aos as atuais, influências no setor saúde e na enfermagem. Texto contexto enferm eriódico na internet]. 2006;15(3):508-514. Disponível em: p: / / www.scielo.br/scielo.php?script=sci_arttext\&pid=S010407072006000300017\& =en. http://dx.doi.org/10.1590/S010407072006000300017. 


\section{ciência}

15. Kurgant P. Administração em enfermagem. São Paulo (SP): EPU; 1991.

16. Figueiredo JO, Albertin AL. Uma análise na relação médico-paciente frente aos recursos das tecnologias da informação. RAI-Revista de Administração e Inovação [ periódico na internet] 2014;11(2):132-153 . Disponível em: < http://www.redalyc.org/pdf/973/97331343008.pdf $>$.

17. Collet N, Gomes ELR, Mishima SM. Método funcional na administração em enfermagem: relato de experiência. Revista Bras. Enferm. 1994, 47 (3): 258-64. 